\title{
Stability Analysis of the Kalman Predictor
}

\author{
Qinghua Zhang a and Liangquan Zhang ${ }^{\mathrm{b}}$ \\ anria, Ifsttar, Université de Rennes, Campus de Beaulieu, 35042 Rennes Cedex, France. Email: \\ qinghua.zhang@inria.fr. \\ ${ }^{\mathrm{b}}$ School of Science, Beijing University of Posts and Telecommunications, Beijing 100876, China. Email: \\ xiaoquan51011@163.com.
}

\author{
ARTICLE HISTORY \\ Compiled May 7, 2019
}

\begin{abstract}
The stability of the Kalman filter, though less often mentioned than the optimality in the recent literature, is a crucial property for real time applications. The purpose of this paper is to complete the classical stability analysis of the Kalman filter for general time varying systems. A proof of the stability of the one step ahead predictor, which is embedded in the Kalman filter, is presented in this paper, whereas the classical results were focused on the stability of the filter. The predictor stability is particularly important for linear parameter varying (LPV) system identification by means of prediction error minimization.
\end{abstract}

\section{KEYWORDS}

Kalman filter, Stability, Boundedness, Prediction error minimization.

\section{Introduction}

The Kalman filter is widely applied in various fields, mainly due to its well known optimal properties and numerical efficiency (Jazwinski, 1970; Anderson and Moore, 1979; Zarchan and Musof, 2005; Kim, 2011; Grewal and Andrews, 2015). Another important property of the Kalman filter, its stability, is less often mentioned in the recent literature. As a recursive algorithm, often implemented for real time applications, it is important to ensure that the computed variables remain bounded, and the recursion dynamics are stable. Hopefully, this paper will contribute to popularize issues related to Kalman filter stability, in addition to completing classical results.

In the classical literature on Kalman filter, the stability property was first studied in Kalman's pioneering work (Kalman, 1963), for the continuous time filter. The discrete time case was then addressed in (Deyst Jr and Price, 1968; Jazwinski, 1970; Deyst Jr, 1973). In these results, the stability of the Kalman filter is essentially based on the uniform complete observability $\left(\mathrm{UCO}^{1}\right)$ and the uniform complete controllability (UCC) of the underlying statespace system. In both the continuous time case and the discrete time case, these results involve reversing the state equation. In the discrete time case, it implies the extra assumption that the state transition matrix is invertible. This invertibility requirement was considered artificial, but it was not easy to relax it due to technical difficulties in the stability analysis. About a decade later, an important improvement was published in (Moore and Anderson, 1980), with a new proof without inverting the state transition matrix.

Liangquan Zhang acknowledges the financial support partly by the National Nature Science Foundation of China (Grant No. 11701040, 11871010, 61871058 \& 61603049) and Innovation Foundation of BUPT for Youth (No. 500417024 \& 505018304).

${ }^{1}$ In this paper the abbreviation UCO will be used either as a noun for "uniform complete observability" or as an adjectif for "uniformly completely observable". The similar abbreviation UCC will be used in the same way. 
Relaxing the invertibility of state transition matrices is particularly important for discrete time linear parameter varying (LPV) systems (Tóth, 2010), though it is of limited interest for linear time invariant (LTI) systems. In an LPV system, the state transition matrix depends on a scheduling variable that evolves in real time, and it may not be possible to guarantee its invertibility in advance. Moreover, even in the case of invertible state transition matrix, it may be difficult to guarantee an upper bound of its inverse, which was also required in (Deyst Jr and Price, 1968; Jazwinski, 1970; Deyst Jr, 1973). In these classical results, as well as in the present paper, linear time varying (LTV) systems are considered, including LPV systems.

The purpose of the present paper is to complete the results of (Moore and Anderson, 1980) with a direct proof of the stability of the Kalman predictor. The Kalman filter algorithm is usually presented with a state prediction step and a filtering step (state update). In (Moore and Anderson, 1980), the exponential stability of the filter is directly proved, whereas the stability of the predictor is indirectly considered through the stability of the filter, based on a time varying and possibly singular linear transformation. In this paper, a direct proof of the predictor stability will be presented, based on a Lyapunov function ensuring the exponential stability of the predictor error dynamics. The availability of a Lyapunov function is important in some analyses, for example, when a state estimator is extended to an adaptive estimator (Zhang and Besançon, 2008). The indirect proof presented in (Moore and Anderson, 1980) does not provide a Lyapunov function of the predictor error dynamics when the linear transformation relating the filter to the predictor is singular. The difficulty for transforming Lyapunov functions after a singular state transformation is highlighted in (Moore and Anderson, 1980), Section 5, after the proof of Theorem 3.1.

The stability of the Kalman predictor is important for LPV system identification based on the prediction error method (PEM) (Ljung, 1999), by ensuring well behaved numerical computation of prediction errors during their minimization. Like in (Moore and Anderson, 1980), discrete time LTV systems will be considered in this paper, without assuming the invertibility of the state transition matrix.

The rest of this paper is organized as follows. The problems studied in this paper are formulated in Section 2. Some preliminary results are presented in Section 3. The stability of the Kalman predictor is proved in Section 4. Concluding remarks are drawn in Section 5. Some proofs and lemmas are presented in the appendix.

\section{Problem statement}

After introducing some notations and recalling the Kalman filter, the problems considered in this paper will be formulated.

\subsection{Notations}

In this paper, For any vector $v,\|v\|$ denotes its Euclidean norm. For any matrix $M,\|M\|$ denotes the matrix norm induced by the Euclidean vector norm, which is equal to the largest singular value of $A$. For a symmetric real matrix $A$, the inequality $A>0$ means that $A$ is positive definite, and $A \geq 0$ means positive semidefinite. For two symmetric real positive (semi)definite matrices $A$ and $B$ of the same size, $A>B$ means $A-B>0$, and $A \geq B$ means $A-B \geq 0$. For any positive integer $l$, the $l \times l$ identity matrix is denoted by $I_{l}$. 


\subsection{The Kalman filter}

Consider the LTV system

$$
\begin{aligned}
x_{k+1} & =A_{k} x_{k}+w_{k}, \\
y_{k} & =C_{k} x_{k}+v_{k},
\end{aligned}
$$

where $k$ is the discrete time index, $x_{k} \in \mathbb{R}^{n}$ is the state, $y_{k} \in \mathbb{R}^{m}$ the output, $w_{k} \in \mathbb{R}^{n}$, $v_{k} \in \mathbb{R}^{m}$ are zero mean white noises independent of each other and of the initial state $x_{0}$, $A_{k}, C_{k}$ are matrices of appropriate sizes, and the noise covariance matrices are $\mathrm{E}\left(w_{k} w_{k}^{T}\right)=Q_{k}$, $\mathrm{E}\left(v_{k} v_{k}^{T}\right)=R_{k}$. The initial state $x_{0}$ has its mean value $\mathrm{E}\left(x_{0}\right)=\bar{x}_{0} \in \mathbb{R}^{n}$, and its covariance $\operatorname{cov}\left(x_{0}\right)=\bar{P}_{0} \in \mathbb{R}^{n \times n}$ (a symmetric positive definite matrix).

It is possible to add an input term $B_{k} u_{k}$ into the state equation. As such an input term has no effect on the stability of the Kalman filter, it is omitted in this paper in order to focus on the stability analysis.

The well known Kalman filter consists of the following recursive computations for $k=$ $0,1,2, \ldots$, after the initialization $P_{0 \mid 0}=\bar{P}_{0}$ and $\hat{x}_{0 \mid 0}=\bar{x}_{0}$ :

$$
\begin{aligned}
P_{k+1 \mid k} & =A_{k} P_{k \mid k} A_{k}^{T}+Q_{k} \\
\Sigma_{k+1} & =C_{k+1} P_{k+1 \mid k} C_{k+1}^{T}+R_{k+1} \\
K_{k+1} & =P_{k+1 \mid k} C_{k+1}^{T} \Sigma_{k+1}^{-1} \\
P_{k+1 \mid k+1} & =\left(I_{n}-K_{k+1} C_{k+1}\right) P_{k+1 \mid k} \\
\hat{x}_{k+1 \mid k} & =A_{k} \hat{x}_{k \mid k} \\
\tilde{y}_{k+1} & =y_{k+1}-C_{k+1} \hat{x}_{k+1 \mid k} \\
\hat{x}_{k+1 \mid k+1} & =\hat{x}_{k+1 \mid k}+K_{k+1} \tilde{y}_{k+1} .
\end{aligned}
$$

\subsection{Focus of this paper and relationship with existing results}

The purpose of this paper is to study the stability of the Kalman filter, with a particular focus on the error dynamics of the one step ahead prediction.

In the early discrete time Kalman filter stability analysis (Deyst Jr and Price, 1968; Jazwinski, 1970; Deyst Jr, 1973), one of the essential assumptions was the existence and the boundedness of the matrix inverse $A_{k}^{-1}$, so that the state equation (1a) can be reversed in time as

$$
x_{k}=A_{k}^{-1} x_{k+1}-A_{k}^{-1} w_{k} .
$$

This invertibility requirement was later relaxed in (Moore and Anderson, 1980), as an important progress in the Kalman filter stability analysis. Technically, it is indeed much more difficult to analyze the stability of the Kalman filter when $A_{k}$ is not invertible. This fact will be highlighted in this paper (see Remark 2).

The present paper aims at completing the results of (Moore and Anderson, 1980) in the following aspect. The Kalman filter algorithm is usually decomposed into a prediction step and a filter (update) step, as expressed in equations (2e) and (2g), respectively. Let $z_{k \mid k-1}$ and $z_{k \mid k}$ denote respectively the mathematical expectation of the one step ahead state prediction error and the filtered (updated) state estimation error, i.e.,

$$
\begin{aligned}
z_{k \mid k-1} & \triangleq \mathrm{E}\left[x_{k}-\hat{x}_{k \mid k-1}\right] \\
z_{k \mid k} & \triangleq \mathrm{E}\left[x_{k}-\hat{x}_{k \mid k}\right] .
\end{aligned}
$$


The filter error dynamics stability (behavior of $z_{k \mid k}$ ) was directly analyzed in (Moore and Anderson, 1980), whereas the predictor error dynamics stability (behavior of $z_{k \mid k-1}$ ) was indirectly considered, based on the fact that $z_{k \mid k}$ and $z_{k \mid k-1}$ are related by $A_{k}$, which is a time varying and possibly singular matrix (see Lemma 6.3 of the cited paper). In the present paper, a direct proof of the predictor stability will be presented. This proof will be based on new technical lemmas, which are different from those of (Moore and Anderson, 1980), because of essential differences between the two error dynamics.

The stability of the predictor is important for LPV system identification based on the prediction error method (PEM) (Ljung, 1999). Like in (Moore and Anderson, 1980), discrete time LTV systems will be considered, without assuming the invertibility of the state transition matrix.

\section{Assumptions and preliminary results}

In this section, the assumptions at the basis of the Kalman filter stability analysis will be first stated, before recalling and completing important lemmas about covariance boundedness, essentially following (Moore and Anderson, 1980).

\subsection{Assumptions}

For any pair of integers $l>k \geq 0$, the state transition matrix $\Phi_{l \mid k}$ is defined as

$$
\begin{aligned}
\Phi_{k \mid k} & =I_{n} \\
\Phi_{l \mid k} & =A_{l-1} \cdots A_{k} .
\end{aligned}
$$

In this paper $\Phi_{l \mid k}$ is not defined for $l<k$, because it would involve the inverse of $A_{k}$, which may be singular. Recall that in (Deyst Jr and Price, 1968; Jazwinski, 1970; Deyst Jr, 1973) it was defined as

$$
\Phi_{l \mid k}=A_{l}^{-1} A_{l+1}^{-1} \cdots A_{k-1}^{-1}, \text { for } l<k .
$$

This case is not required in the present paper.

Assumption 1 (Boundedness). There exist positive constants $\bar{a}, \bar{c}, \bar{q}, \underline{r}, \bar{r}$, such that, for all $k=0,1,2, \ldots$,

$$
\begin{aligned}
\left\|A_{k}\right\| & \leq \bar{a} \\
\left\|C_{k}\right\| & \leq \bar{c} \\
Q_{k} & \leq \bar{q} I_{n} \\
\underline{r} I_{m} \leq R_{k} & \leq \bar{r} I_{m} .
\end{aligned}
$$

Assumption 2 (UCO). The matrix pair $\left(A_{k}, C_{k}\right)$ is uniformly completely observable, in the sense that there exist two positive constants $\alpha_{2}>\alpha_{1}>0$ and an integer $h>0$ such that, for all integer $k \geq 0$,

$$
\alpha_{1} I_{n} \leq \sum_{i=k}^{k+h-1} \Phi_{i \mid k}^{T} C_{i}^{T} C_{i} \Phi_{i \mid k} \leq \alpha_{2} I_{n}
$$

Assumption 3 (UCC). The matrix pair $\left(A_{k}, Q_{k}^{\frac{1}{2}}\right)$ is uniformly completely controllable (UCC) in the sense that there exist two positive constants $\beta_{2}>\beta_{1}>0$ and a positive integer $s$ such 
that, for all $k=s, s+1, \ldots$,

$$
\beta_{1} I_{n} \leq \sum_{i=k-s+1}^{k} \Phi_{k+1 \mid i+1} Q_{i} \Phi_{k+1 \mid i+1}^{T} \leq \beta_{2} I_{n}
$$

\subsection{Error dynamics}

Let $\tilde{x}_{k \mid k-1}$ and $\tilde{x}_{k \mid k}$ denote respectively the state prediction error and the filter error (also known as update error), defined as

$$
\begin{gathered}
\tilde{x}_{k \mid k-1} \triangleq x_{k}-\hat{x}_{k \mid k-1} \\
\tilde{x}_{k \mid k} \triangleq x_{k}-\hat{x}_{k \mid k} .
\end{gathered}
$$

It is then derived from (1) and (2) that

$$
\begin{aligned}
\tilde{x}_{k+1 \mid k} & =A_{k} \tilde{x}_{k \mid k}+w_{k} \\
\tilde{x}_{k+1 \mid k+1} & =\left(I_{n}-K_{k+1} C_{k+1}\right) \tilde{x}_{k+1 \mid k}-K_{k+1} v_{k+1} .
\end{aligned}
$$

Combining (13) and (14) yields the predictor error dynamics equation

$$
\tilde{x}_{k+1 \mid k}=A_{k}\left(I_{n}-K_{k} C_{k}\right) \tilde{x}_{k \mid k-1}-A_{k} K_{k} v_{k}+w_{k},
$$

or alternatively, the filter error dynamics equation

$$
\tilde{x}_{k+1 \mid k+1}=\left(I_{n}-K_{k+1} C_{k+1}\right) A_{k} \tilde{x}_{k \mid k}+\left(I_{n}-K_{k+1} C_{k+1}\right) w_{k}-K_{k+1} v_{k+1} .
$$

The mathematical expectation of the prediction error

$$
z_{k \mid k-1}=\mathrm{E}\left[\tilde{x}_{k \mid k-1}\right]
$$

then satisfies

$$
z_{k+1 \mid k}=A_{k}\left(I_{n}-K_{k} C_{k}\right) z_{k \mid k-1},
$$

which coincides with the homogeneous part of (15).

Analogously, the mathematical expectation of the filter error

$$
z_{k \mid k}=\mathrm{E}\left[\tilde{x}_{k \mid k}\right]
$$

satisfies

$$
z_{k+1 \mid k+1}=\left(I_{n}-K_{k+1} C_{k+1}\right) A_{k} z_{k \mid k}
$$

which coincides with the homogeneous part of (16).

\subsection{Error covariances}

As a recursive algorithm, it is important to ensure that the predictor error covariance $P_{k+1 \mid k}$ and the filter error covariance $P_{k \mid k}$ computed in (2a) and in (2d) are both bounded. 
Lemma 1. Under Assumptions 1 and 2, the covariance matrices $P_{k+1 \mid k}$ and $P_{k \mid k}$ are upper bounded, i.e., there exist positive constants $\rho_{1}, \rho_{2}$ such that, for all $k=0,1,2, \ldots$,

$$
\begin{aligned}
P_{k+1 \mid k} & \leq \rho_{1} I_{n} \\
P_{k \mid k} & \leq \rho_{2} I_{n} .
\end{aligned}
$$

For a proof of these results see Lemma 6.1 in (Moore and Anderson, 1980). A trivial corollary of this lemma is that the Kalman gain $K_{k}$ is also bounded. For the stability analysis of the next section, it is important to ensure that $P_{k+1 \mid k}$ and $P_{k \mid k}$ have each a strictly positive lower bound.

Lemma 2. Under Assumptions 1-3, $P_{k \mid k}$ has a strictly positive lower bound, i.e., there exists a positive constant $\rho_{3}$ such that, for all $k>\max (h, s)$, with the integers $h$ and $s$ as those involved in Assumptions 2 and 3,

$$
P_{k \mid k} \geq \rho_{3} I_{n}
$$

This result was established as Lemma 6.2 in (Moore and Anderson, 1980). The lower bound of $P_{k+1 \mid k}$ was not investigated in (Moore and Anderson, 1980), where only the stability of the filter error dynamics was directly considered. In the present paper, in order to analyze the stability of the predictor error dynamics, it is necessary to complete the above result with the following lemma.

Lemma 3. Under Assumptions 1-3, $P_{k+1 \mid k}$ has a strictly positive lower bound, i.e., for all $k>\max (h, s)$, with the integers $h$ and $s$ as those involved in Assumptions 2 and 3,

$$
P_{k+1 \mid k} \geq \rho_{3} I_{n},
$$

where $\rho_{3}$ is the same constant as in Lemma 2.

In the classical results (Deyst Jr and Price, 1968; Jazwinski, 1970; Deyst Jr, 1973) where it was assumed that $A_{k}$ is invertible and $A_{k}^{-1}$ is bounded, a lower bound of $P_{k+1 \mid k}$ was trivially derived from the lower bound of $P_{k \mid k}$ through (2a). In this paper, $A_{k}$ can be singular, it is thus less obvious to establish a lower bound of $P_{k+1 \mid k}$.

Proof of Lemma 3. As a covariance matrix, $P_{k+1 \mid k}$ is always symmetric and positive semidefinite. This fact can also be seen from (2a), where the two terms at the right hand side are both symmetric and positive semidefinite.

Combine (2d) and (2c) to obtain

$$
\begin{aligned}
P_{k+1 \mid k+1} & =P_{k+1 \mid k}-K_{k+1} C_{k+1} P_{k+1 \mid k} \\
& =P_{k+1 \mid k}-P_{k+1 \mid k} C_{k+1}^{T} \Sigma_{k+1}^{-1} C_{k+1} P_{k+1 \mid k} .
\end{aligned}
$$

According to Assumption 1, $R_{k}$ satisfies $0<\underline{r} I_{m} \leq R_{k} \leq \bar{r} I_{m}$. Hence the innovation covariance matrix $\Sigma_{k+1}$ computed in (2b) is positive definite and $\Sigma_{k+1}^{-1}$ is bounded. Therefore,

$$
P_{k+1 \mid k} C_{k+1}^{T} \Sigma_{k+1}^{-1} C_{k+1} P_{k+1 \mid k} \geq 0 .
$$

It then follows from (26) that

$$
\begin{aligned}
P_{k+1 \mid k} & =P_{k+1 \mid k+1}+P_{k+1 \mid k} C_{k+1}^{T} \Sigma_{k+1}^{-1} C_{k+1} P_{k+1 \mid k} \\
& \geq P_{k+1 \mid k+1} \\
& \geq \rho_{3} I_{n}
\end{aligned}
$$


where the last inequality follows from Lemma 2.

\section{Predictor error dynamics stability}

In this section, two important lemmas are first presented before the stability analysis.

\subsection{Auxiliary results}

Lemma 4. Let $A, P, Q$ be square real matrices of the same size, $A$ is possibly singular, $P, Q$ are symmetric, $P>0, Q \geq 0$ and $\left(A P A^{T}+Q\right)>0$. Then

$$
A^{T}\left(A P A^{T}+Q\right)^{-1} A \leq P^{-1}
$$

A proof of this lemma is presented in Appendix B at the end of this paper.

Remark 1. In this lemma, $A$ and $Q$ may be singular, but $\left(A P A^{T}+Q\right)>0$ is assumed. In the case of invertible $A$, the proof of Lemma 4 is quite trivial. However, the proof is non trivial for a singular matrix $A$. This lemma will play an important role in the stability proof without inverting the state transition matrix.

The matrix

$$
\bar{A}_{k} \triangleq A_{k}\left(I-K_{k} C_{k}\right)
$$

characterizes the behavior of the predictor error equation (15), or of its homogenous part (18). The corresponding state transition matrix is

$$
\bar{\Phi}_{l \mid k} \triangleq \bar{A}_{l-1} \cdot \bar{A}_{l-2} \cdots \bar{A}_{k}
$$

for integers $l>k \geq 0$, and $\bar{\Phi}_{k \mid k} \triangleq I_{n}$. This state transition matrix is not defined for $l<k$.

The following lemma states that, essentially, if the matrix pair $\left(A_{k}, C_{k}\right)$ is $\mathrm{UCO}$, then so is $\left(\bar{A}_{k}, C_{k}\right)$. For noise-free systems (observability is indeed a property concerning the deterministic part of the system only), $\bar{A}_{k} x_{k}=A_{k}\left(I_{n}-K_{k} C_{k}\right) x_{k}=A_{k} x_{k}-K_{k} y_{k}$, then this lemma means that the output feedback term $K_{k} y_{k}$ preserves the observability of the system. This result remains true if the optimal gain $K_{k}$ is replaced by any bounded non optimal gain.

Lemma 5. Under Assumptions 1-2, notably Assumption 2 on the UCO of $\left(A_{k}, C_{k}\right)$ expressed by the two inequalities (9), there exist two positive constants $\bar{\alpha}_{2}>\bar{\alpha}_{1}>0$ such that, for all integer $k \geq 0$,

$$
\bar{\alpha}_{1} I \leq \sum_{i=k}^{k+h-1} \bar{\Phi}_{i \mid k}^{T} C_{i}^{T} C_{i} \bar{\Phi}_{i \mid k} \leq \bar{\alpha}_{2} I
$$

where $\bar{\Phi}_{i \mid k}$ as defined in (33) is the state transition matrix corresponding to $\bar{A}_{k}=A_{k}\left(I-K_{k} C_{k}\right)$, and $h$ is the same integer as in (9).

This lemma is equivalent to the Lemma A.1 in the appendix of (Moore and Anderson, 1980), where hints for its proof are also given. The proof for a slightly different lemma can be found in (Zhang, 2017), with a UCO definition equivalent to that of (Jazwinski, 1970), but different from (Moore and Anderson, 1980). Some related results for continuous time systems are available in (Sastry and Bodson, 1989; Ioannou and Sun, 1996; Zhang and Zhang, 2015). 


\subsection{Stability analysis}

The following stability result states that, under Assumptions 1-3 (essentially the boundedness of system matrices, the UCO and UCC), the predictor error dynamics of the Kalman filter are exponentially stable. As the stability of the predictor error dynamics concerns only the homogenous part of the error equation (15), the corresponding homogeneous equation (18) will be referred to as the predictor error dynamics equation in the following analysis.

Theorem 6. Under Assumptions 1-3, the prediction error dynamics equation (18), namely

$$
z_{k+1 \mid k}=A_{k}\left(I_{n}-K_{k} C_{k}\right) z_{k \mid k-1}
$$

is exponentially stable.

Proof. According to Lemmas 1 and $3, P_{k \mid k-1}$ has a finite upper bound and a strictly positive lower bound.

Define the Lyapunov function candidate

$$
V\left(z_{k \mid k-1}, P_{k \mid k-1}\right) \triangleq z_{k \mid k-1}^{T} P_{k \mid k-1}^{-1} z_{k \mid k-1},
$$

then

$$
\begin{aligned}
V\left(z_{k+1 \mid k}, P_{k+1 \mid k}\right) & =z_{k+1 \mid k}^{T} P_{k+1 \mid k}^{-1} z_{k+1 \mid k} \\
& =\left[A_{k}\left(I_{n}-K_{k} C_{k}\right) z_{k \mid k-1}\right]^{T} P_{k+1 \mid k}^{-1}\left[A_{k}\left(I_{n}-K_{k} C_{k}\right) z_{k \mid k-1}\right] \\
& =z_{k \mid k-1}^{T} \Xi_{k} z_{k \mid k-1}
\end{aligned}
$$

with

$$
\Xi_{k} \triangleq\left(I_{n}-K_{k} C_{k}\right)^{T} A_{k}^{T} P_{k+1 \mid k}^{-1} A_{k}\left(I_{n}-K_{k} C_{k}\right)
$$

According to Lemma $3, P_{k+1 \mid k}>0$. Then by recalling equation (2a) and by applying Lemma 4 , it yields

$$
A_{k}^{T} P_{k+1 \mid k}^{-1} A_{k}=A_{k}^{T}\left(A_{k} P_{k \mid k} A_{k}^{T}+Q_{k}\right)^{-1} A_{k} \leq P_{k \mid k}^{-1}
$$

Therefore,

$$
\Xi_{k} \leq\left(I_{n}-K_{k} C_{k}\right)^{T} P_{k \mid k}^{-1}\left(I_{n}-K_{k} C_{k}\right)
$$

Rewrite (2d) as

$$
P_{k \mid k}^{-1}\left(I_{n}-K_{k} C_{k}\right)=P_{k \mid k-1}^{-1}
$$

and substitute it into (42), then

$$
\begin{aligned}
\Xi_{k} & \leq\left(I_{n}-K_{k} C_{k}\right)^{T} P_{k \mid k-1}^{-1} \\
& =P_{k \mid k-1}^{-1}-C_{k}^{T} K_{k}^{T} P_{k \mid k-1}^{-1}
\end{aligned}
$$

Reformulate (2c) as

$$
K_{k}^{T} P_{k \mid k-1}^{-1}=\Sigma_{k}^{-1} C_{k},
$$


then

$$
\Xi_{k} \leq P_{k \mid k-1}^{-1}-C_{k}^{T} \Sigma_{k}^{-1} C_{k}
$$

By recalling (39),

$$
\begin{aligned}
V\left(z_{k+1 \mid k}, P_{k+1 \mid k}\right) & =z_{k \mid k-1}^{T} \Xi_{k} z_{k \mid k-1} \\
& \leq z_{k \mid k-1}^{T} P_{k \mid k-1}^{-1} z_{k \mid k-1}-z_{k \mid k-1}^{T} C_{k}^{T} \Sigma_{k}^{-1} C_{k} z_{k \mid k-1} \\
& =V\left(z_{k \mid k-1}, P_{k \mid k-1}\right)-z_{k \mid k-1}^{T} C_{k}^{T} \Sigma_{k}^{-1} C_{k} z_{k \mid k-1}
\end{aligned}
$$

hence

$$
V\left(z_{k+1 \mid k}, P_{k+1 \mid k}\right)-V\left(z_{k \mid k-1}, P_{k \mid k-1}\right) \leq-z_{k \mid k-1}^{T} C_{k}^{T} \Sigma_{k}^{-1} C_{k} z_{k \mid k-1} .
$$

According to $(2 \mathrm{~b})$,

$$
\left\|\Sigma_{k}\right\|=\left\|C_{k} P_{k \mid k-1} C_{k}^{T}+R_{k}\right\| .
$$

By applying the triangular inequality and the sub-multiplicative inequality of the considered matrix norm (recalled as Lemma 12 in Appendix A),

$$
\begin{aligned}
\left\|\Sigma_{k}\right\| & \leq\left\|C_{k}\right\|\left\|P_{k \mid k-1}\right\|\left\|C_{k}\right\|+\left\|R_{k}\right\| \\
& \leq \bar{c}^{2} \rho_{1}+\bar{r}
\end{aligned}
$$

then (see Lemma 13 in Appendix A),

$$
\Sigma_{k} \leq\left(\bar{c}^{2} \rho_{1}+\bar{r}\right) I_{m}
$$

It then follows from (51) that

$$
V\left(z_{k+1 \mid k}, P_{k+1 \mid k}\right)-V\left(z_{k \mid k-1}, P_{k \mid k-1}\right) \leq-\left(\bar{c}^{2} \rho_{1}+\bar{r}\right)^{-1} z_{k \mid k-1}^{T} C_{k}^{T} C_{k} z_{k \mid k-1} .
$$

Combine the instances of the last inequality at the time instants $k, k+1, \ldots, k+h-1$, then

$$
V\left(z_{k+h}, P_{k+h \mid k+h-1}\right)-V\left(z_{k \mid k-1}, P_{k \mid k-1}\right) \leq-\left(\bar{c}^{2} \rho_{1}+\bar{r}\right)^{-1} \sum_{s=k}^{k+h-1} z_{s \mid s-1}^{T} C_{s}^{T} C_{s} z_{s \mid s-1} .
$$

Notice that $z_{s \mid s-1}$ satisfies (35), with the associated state transition matrix $\bar{\Phi}_{l \mid k}$ as defined in (33) and (32). It then follows from the definition of $\bar{\Phi}_{l \mid k}$ and equation (35) that

$$
z_{s \mid s-1}=\bar{\Phi}_{s \mid k} z_{k \mid k-1} \text {. }
$$

Then inequality (57) is continued as

$$
\begin{aligned}
V\left(z_{k+h}, P_{k+h \mid k+h-1}\right)-V\left(z_{k \mid k-1}, P_{k \mid k-1}\right) & \leq-\left(\bar{c}^{2} \rho_{1}+\bar{r}\right)^{-1} z_{k \mid k-1}^{T}\left(\sum_{s=k}^{k+h-1} \bar{\Phi}_{s \mid k}^{T} C_{s}^{T} C_{s} \bar{\Phi}_{s \mid k}\right) z_{k \mid k-1} \\
& \leq-\left(\bar{c}^{2} \rho_{1}+\bar{r}\right)^{-1} \bar{\alpha}_{1} z_{k \mid k-1}^{T} z_{k \mid k-1}
\end{aligned}
$$

where $\bar{\alpha}_{1}$ is as in (34), based on Lemma 5 . 
A lower bound of $P_{k \mid k-1}$ has been established in Lemma 3, thus $P_{k \mid k-1} \geq \rho_{3} I_{n}$. Then

$$
\begin{aligned}
V\left(z_{k+h}, P_{k+h \mid k+h-1}\right)-V\left(z_{k \mid k-1}, P_{k \mid k-1}\right) & \leq-\left(\bar{c}^{2} \rho_{1}+\bar{r}\right)^{-1} \bar{\alpha}_{1} \rho_{3} z_{k \mid k-1}^{T} P_{k \mid k-1}^{-1} z_{k \mid k-1} \\
& =-\rho_{4} V\left(z_{k \mid k-1}, P_{k \mid k-1}\right) .
\end{aligned}
$$

with

$$
\rho_{4} \triangleq\left(\bar{c}^{2} \rho_{1}+\bar{r}\right)^{-1} \bar{\alpha}_{1} \rho_{3}>0
$$

Therefore,

$$
V\left(z_{k+h}, P_{k+h \mid k+h-1}\right) \leq\left(1-\rho_{4}\right) V\left(z_{k \mid k-1}, P_{k \mid k-1}\right) .
$$

By definition $V\left(z_{k+h}, P_{k+h \mid k+h-1}\right) \geq 0$ and $\rho_{4}>0$, then

$$
0 \leq\left(1-\rho_{4}\right)<1
$$

Then, for all $k>h$,

$$
V\left(z_{k \mid k-1}, P_{k \mid k-1}\right) \leq\left(1-\rho_{4}\right)^{\left.L^{\frac{k-1}{h}}\right\rfloor} V\left(z_{1 \mid 0}, P_{1 \mid 0}\right)
$$

where $\lfloor x\rfloor$ denotes the largest integer not larger than $x$. Therefore,

$$
V\left(z_{k \mid k-1}, P_{k \mid k-1}\right) \leq\left[\left(1-\rho_{4}\right)^{\frac{1}{h}}\right]^{k-h-1} V\left(z_{1 \mid 0}, P_{1 \mid 0}\right),
$$

hence $V\left(z_{k \mid k-1}, P_{k \mid k-1}\right)$ tends exponentially to zero. So does $z_{k \mid k-1}$.

Remark 2. In this proof, the step from (40) to (42) was based on the important inequality

$$
A_{k}^{T}\left(A_{k} P_{k \mid k} A_{k}^{T}+Q_{k}\right)^{-1} A_{k} \leq P_{k \mid k}^{-1},
$$

which would be relatively trivial if $A_{k}$ was assumed invertible. This step is indeed a major difficulty when $A_{k}$ is possibly singular. Lemma 4 played an important role to overcome this difficulty.

To our knowledge, Theorem 6 is the first direct result about the stability of the one step ahead predictor embedded in the Kalman filter, for systems with possibly non invertible state transition matrices. The classical results reported in (Deyst Jr and Price, 1968; Jazwinski, 1970; Deyst Jr, 1973; Moore and Anderson, 1980) were focused on the stability of the Kalman filter. This result on the predictor stability has an important impact on the development of methods for LPV system identification. As the predictor stability has been established regardless of the stability of the underlying dynamic system, an LPV model can be estimated by minimizing the prediction error, no matter if the system being identified is stable or not.

\section{Conclusion}

Among the properties of the Kalman filter, the stability is of crucial importance for real time applications. In practice, the optimality of the Kalman filter may be approximative due to uncertainties in model parameters, but it is important to formally establish its stability. The earliest stability analysis for discrete time Kalman filters assumed the invertibility of the state transition matrix (Deyst Jr and Price, 1968; Jazwinski, 1970; Deyst Jr, 1973). This limitation 
was later relaxed in (Moore and Anderson, 1980). In the present paper, these classical results are completed by a direct proof of the one step ahead predictor stability, which is of particular importance for the current development of LPV system identification based on prediction error minimization.

\section{Appendix A. Some lemmas and proofs}

The purpose of this section is to prove Lemma 4. To this end, let us first recall some well known results.

Lemma 7. Let $A, B, C, D$ be matrices of appropriate sizes, and assume that the matrix inverses involved in the following formula exist, then

$$
\left[\begin{array}{ll}
A & B \\
C & D
\end{array}\right]^{-1}=\left[\begin{array}{cc}
\left(A-B D^{-1} C\right)^{-1} & -A^{-1} B\left(D-C A^{-1} B\right)^{-1} \\
-D^{-1} C\left(A-B D^{-1} C\right)^{-1} & \left(D-C A^{-1} B\right)^{-1}
\end{array}\right] .
$$

This well known result can be proved by simply computing the matrix product leading to an identity matrix.

Lemma 8. Suppose that $A, B$ are symmetric positive definite matrices. Then $0<A<B \Longrightarrow$ $A^{-1}>B^{-1}>0$, and similarly, $0<A \leq B \Longrightarrow A^{-1} \geq B^{-1}>0$.

Proof. The proof of this seemingly trivial result is not really trivial.

$$
\begin{aligned}
& 0<A<B \Rightarrow I<A^{-\frac{1}{2}} B^{\frac{1}{2}} B^{\frac{1}{2}} A^{-\frac{1}{2}} \Rightarrow\left(B^{\frac{1}{2}} A^{-\frac{1}{2}}\right)^{-1}\left(A^{-\frac{1}{2}} B^{\frac{1}{2}}\right)^{-1}<I \Rightarrow A^{\frac{1}{2}} B^{-1} A^{\frac{1}{2}}<I \\
& \Rightarrow B^{-1}<A^{-1} .
\end{aligned}
$$

The proof for the semi-definite case is similar.

Lemma 9. Let $\Omega>0$ and $\Psi>0$ be symmetric real matrices, $\Gamma$ is a real matrix of appropriate size,

$$
P=\left[\begin{array}{cc}
\Omega & \Gamma \\
\Gamma^{T} & \Psi
\end{array}\right]
$$

Under the above assumptions, $P>0$ if and only if $\Omega-\Gamma \Psi^{-1} \Gamma^{T}>0$.

This lemma is well known, but its "semi-definite" variant stated below is less well known.

Lemma 10. $\Omega \geq 0$ and $\Psi>0$ are symmetric real matrices, $\Gamma$ is a real matrix of appropriate size,

$$
P=\left[\begin{array}{cc}
\Omega & \Gamma \\
\Gamma^{T} & \Psi
\end{array}\right]
$$

Under the above assumptions, $P \geq 0$ if and only if $\Omega-\Gamma \Psi^{-1} \Gamma^{T} \geq 0$. 
Proof. The proof below is for Lemma 10, whereas Lemma 9 can be proved similarly. For sufficiency first. For two real vectors $x, y$ of appropriate dimensions, define

$$
\begin{aligned}
V(x, y) & \triangleq\left[x^{T}, y^{T}\right] P\left[x^{T}, y^{T}\right]^{T} \\
& =x^{T} \Omega x+y^{T} \Psi y+2 x^{T} \Gamma y \\
& =\left(y+\Psi^{-1} \Gamma^{T} x\right)^{T} \Psi\left(y+\Psi^{-1} \Gamma^{T} x\right)+x^{T}\left(\Omega-\Gamma \Psi^{-1} \Gamma^{T}\right) x
\end{aligned}
$$

The first term in this expression is non negative, therefore, for any given $x, V(x, y)$ reaches its minimum with $y=-\Psi^{-1} \Gamma^{T} x$, and

$$
\min _{y} V(x, y)=x^{T}\left(\Omega-\Gamma \Psi^{-1} \Gamma^{T}\right) x
$$

It is assumed that $\Omega-\Gamma \Psi^{-1} \Gamma^{T} \geq 0$, then for any $x$ and $y$,

$$
V(x, y) \geq \min _{y} V(x, y)=x^{T}\left(\Omega-\Gamma \Psi^{-1} \Gamma^{T}\right) x \geq 0,
$$

hence $P \geq 0$ is proved.

Now for necessity. $P \geq 0$ implies $V(x, y) \geq 0$ for all $x, y$.

For any given $x$, consider $y=-\Psi^{-1} \Gamma^{T} x$. With this special choice of $y, V(x, y) \geq 0$ holds too.

$$
\begin{aligned}
V(x, y) & =x^{T} \Omega x+y^{T} \Psi y+2 x^{T} \Gamma y \\
& =x^{T} \Omega x+x^{T} \Gamma \Psi^{-1} \Psi \Psi^{-1} \Gamma^{T} x-2 x^{T} \Gamma \Psi^{-1} \Gamma^{T} x \\
& =x^{T}\left(\Omega-\Gamma \Psi^{-1} \Gamma^{T}\right) x
\end{aligned}
$$

hence $x^{T}\left(\Omega-\Gamma \Psi^{-1} \Gamma^{T}\right) x \geq 0$ for any given $x$. Therefore $\Omega-\Gamma \Psi^{-1} \Gamma^{T} \geq 0$.

Lemma 11. For any real symmetric positive definite matrix

$$
P=\left[\begin{array}{cc}
\Omega & \Gamma \\
\Gamma^{T} & \Psi
\end{array}\right]>0
$$

the inequatity

$$
\left[\begin{array}{cc}
\Omega^{-1} & 0 \\
0 & 0
\end{array}\right] \leq P^{-1}
$$

holds.

Remark: the assumption $P>0$ implies $\Omega>0$.

Proof. Apply Lemma 7 to $P$, then

$$
P^{-1}-\left[\begin{array}{cc}
\Omega^{-1} & 0 \\
0 & 0
\end{array}\right]=\left[\begin{array}{cc}
\left(\Omega-\Gamma \Psi^{-1} \Gamma^{T}\right)^{-1}-\Omega^{-1} & -\Omega^{-1} \Gamma\left(\Psi-\Gamma^{T} \Omega^{-1} \Gamma\right)^{-1} \\
-\Psi^{-1} \Gamma^{T}\left(\Omega-\Gamma \Psi^{-1} \Gamma^{T}\right)^{-1} & \left(\Psi-\Gamma^{T} \Omega^{-1} \Gamma\right)^{-1}
\end{array}\right] .
$$

Remark: this result is indeed a symmetric matrix, though it is not obvious.

According to Lemma $9, P>0$ implies $\left(\Omega-\Gamma \Psi^{-1} \Gamma^{T}\right)>0$. Similarly, $\left(\Psi-\Gamma^{T} \Omega^{-1} \Gamma\right)>0$, then $\left(\Psi-\Gamma^{T} \Omega^{-1} \Gamma\right)^{-1}>0$ 
According to Lemma 8, the inequalities

$$
0<\left(\Omega-\Gamma \Psi^{-1} \Gamma^{T}\right) \leq \Omega
$$

imply

$$
\left(\Omega-\Gamma \Psi^{-1} \Gamma^{T}\right)^{-1} \geq \Omega^{-1}>0,
$$

hence the first diagonal block at the RHS of (A4) satisfies $\left(\Omega-\Gamma \Psi^{-1} \Gamma^{T}\right)^{-1}-\Omega^{-1} \geq 0$.

Now in order to apply Lemma 10 to the matrix at the RHS of (A4), compute

$$
\begin{aligned}
& {\left[\left(\Omega-\Gamma \Psi^{-1} \Gamma^{T}\right)^{-1}-\Omega^{-1}\right]-\left[-\Omega^{-1} \Gamma\left(\Psi-\Gamma^{T} \Omega^{-1} \Gamma\right)^{-1}\right] } \\
& \times\left[\left(\Psi-\Gamma^{T} \Omega^{-1} \Gamma\right)^{-1}\right]^{-1}\left[-\Psi^{-1} \Gamma^{T}\left(\Omega-\Gamma \Psi^{-1} \Gamma^{T}\right)^{-1}\right] \\
= & {\left[\left(\Omega-\Gamma \Psi^{-1} \Gamma^{T}\right)^{-1}-\Omega^{-1}\right]-\left[\Omega^{-1} \Gamma\right]\left[\Psi^{-1} \Gamma^{T}\left(\Omega-\Gamma \Psi^{-1} \Gamma^{T}\right)^{-1}\right] } \\
= & -\Omega^{-1}+\left(I-\Omega^{-1} \Gamma \Psi^{-1} \Gamma^{T}\right)\left(\Omega-\Gamma \Psi^{-1} \Gamma^{T}\right)^{-1} \\
= & -\Omega^{-1}+\Omega^{-1}\left(\Omega-\Gamma \Psi^{-1} \Gamma^{T}\right)\left(\Omega-\Gamma \Psi^{-1} \Gamma^{T}\right)^{-1} \\
= & -\Omega^{-1}+\Omega^{-1} \\
= & 0 .
\end{aligned}
$$

Then Lemma 10 ensures that the RHS of (A4) is positive semi-definite, so is the matrix at the LHS of (A4), hence

$$
\left[\begin{array}{cc}
\Omega^{-1} & 0 \\
0 & 0
\end{array}\right] \leq P^{-1}
$$

Lemma 12. For any two matrices $A \in \mathbb{R}^{m \times n}, B \in \mathbb{R}^{l \times m}$, the inequality $\|B A\| \leq\|B\|\|A\|$ holds, where $\|\cdot\|$ denotes the matrix norm induced by the Euclidean vector norm (as in the whole paper).

Proof. Let $\|x\|$ denote the Euclidean norm of any vector $x \in \mathbb{R}^{n}$. For any matrix $A \in \mathbb{R}^{m \times n}$, its induced norm

$$
\|A\| \triangleq \max _{\|x\|=1}\|A x\|
$$

For any non zero vector $z \in \mathbb{R}^{n}, z /\|z\|$ has a unitary norm, therefore,

$$
\|A\|=\max _{z \neq 0}\left\|A \frac{z}{\|z\|}\right\|=\max _{z \neq 0} \frac{\|A z\|}{\|z\|} \geq \frac{\|A z\|}{\|z\|}
$$

then

$$
\|A z\| \leq\|A\|\|z\|
$$

which holds also trivially for $z=0$. This inequality implies that, for any two matrices $A \in \mathbb{R}^{m \times n}$, $B \in \mathbb{R}^{l \times m}$ and any vector $x \in \mathbb{R}^{n}$,

$$
\|B A x\| \leq\|B\|\|A x\| \leq\|B\|\|A\|\|x\| .
$$


Again by definition of the matrix norm as in (A13),

$$
\|B A\|=\max _{\|x\|=1}\|B A x\| .
$$

Let $x_{*}$ be the vector maximizing $\|B A x\|$ under the constraint $\|x\|=1$, then

$$
\|B A\|=\left\|B A x_{*}\right\| \text { and }\left\|x_{*}\right\|=1 .
$$

Of course, $B, A$ and $x_{*}$ satisfy also (A16), then

$$
\|B A\|=\left\|B A x_{*}\right\| \leq\|B\|\|A\|\left\|x_{*}\right\|=\|B\|\|A\|,
$$

hence Lemma 12 is established.

Lemma 13. For any symmetric positive (semi)definite matrix $A \in \mathbb{R}^{n \times n}$, the inequality $A \leq$ $\|A\| I_{n}$ holds, where $\|\cdot\|$ denotes the matrix norm induced by the Euclidean vector norm, $A \leq B$ means that $B-A$ is a positive semidefinite matrix, and $I_{n}$ denotes the $n \times n$ identity matrix (as in the whole paper).

Proof. The considered matrix norm $\|A\|$ is equal to the largest singular value of $A$, which is also equal to the largest eigenvalue for the symmetric positive (semi)definite matrix $A$. Consider the spectral decomposition

$$
A=U D U^{T}
$$

where $D$ is a diagonal matrix containing the eigenvalues of $A$ (which are all positive or zero, since $A$ is symmetric positive (semi)definite), $U$ is a unitary matrix. Then, for any vector $x \in \mathbb{R}^{n}$,

$$
x^{T} A x=\left(U^{T} x\right)^{T} D\left(U^{T} x\right) \leq \lambda_{\max }\left\|U^{T} x\right\|^{2}
$$

where $\lambda_{\max }$ is the largest eigenvalue of $A$. As $U$ is a unitary matrix, $\left\|U^{T} x\right\|^{2}=x^{T} x$, hence

$$
x^{T} A x \leq \lambda_{\max } x^{T} x .
$$

As mentioned above, the largest eigenvalue $\lambda_{\max }=\|A\|$, then, for any vector $x \in \mathbb{R}^{n}$,

$$
x^{T} A x \leq\|A\| x^{T} I_{n} x,
$$

which means that $A \leq\|A\| I_{n}$.

\section{Appendix B. Proof of Lemma 4}

The proof of this result is trivial in the case of non singular $A$, hence only the case of singular $A$ is considered below.

For a singular $A$, there exists a square real invertible matrix $T$ of the same size such that

$$
A_{T} \triangleq T A T^{-1}=\left[\begin{array}{ll}
\Lambda & 0 \\
0 & 0
\end{array}\right],
$$

where $\Lambda$ is a $r \times r$ invertible matrix, with $r$ equal to the rank of $A$. 
Denote

$$
M \triangleq A^{T}\left(A P A^{T}+Q\right)^{-1} A,
$$

then, replace $A$ with $A=T^{-1} A_{T} T$,

$$
\begin{aligned}
M & =T^{T} A_{T}^{T} T^{-T}\left(T^{-1} A_{T} T P T^{T} A_{T}^{T} T^{-T}+Q\right)^{-1} T^{-1} A_{T} T \\
& =T^{T} A_{T}^{T}\left(A_{T} T P T^{T} A_{T}^{T}+T Q T^{T}\right)^{-1} A_{T} T
\end{aligned}
$$

Let

$$
\begin{gathered}
P_{T} \triangleq T P T^{T} \\
Q_{T} \triangleq T Q T^{T},
\end{gathered}
$$

then

$$
M=T^{T} A_{T}^{T}\left(A_{T} P_{T} A_{T}^{T}+Q_{T}\right)^{-1} A_{T} T
$$

Partition $P_{T}$ and $Q_{T}$ as

$$
P_{T}=\left[\begin{array}{ll}
P_{T}^{(1,1)} & P_{T}^{(1,2)} \\
P_{T}^{(2,1)} & P_{T}^{(2,2)}
\end{array}\right], \quad Q_{T}=\left[\begin{array}{ll}
Q_{T}^{(1,1)} & Q_{T}^{(1,2)} \\
Q_{T}^{(2,1)} & Q_{T}^{(2,2)}
\end{array}\right]
$$

so that $P_{T}^{(1,1)}$ and $Q_{T}^{(1,1)}$ have the same size as the $r \times r$ matrix $\Lambda$.

Due to the block form of $A_{T}$ as in (B1),

$$
A_{T} P_{T} A_{T}^{T}+Q_{T}=\left[\begin{array}{cc}
\Lambda P_{T}^{(1,1)} \Lambda^{T} & 0 \\
0 & 0
\end{array}\right]+Q_{T}=\left[\begin{array}{cc}
\Lambda P_{T}^{(1,1)} \Lambda^{T}+Q_{T}^{(1,1)} & Q_{T}^{(1,2)} \\
Q_{T}^{(2,1)} & Q_{T}^{(2,2)}
\end{array}\right]
$$

It is assumed that $\left(A P A^{T}+Q\right)>0$, therefore,

$$
A_{T} P_{T} A_{T}^{T}+Q_{T}=T\left(A P A^{T}+Q\right) T^{T}>0
$$

This result implies the invertibility of the block $Q_{T}^{(2,2)}$ in (B9), which will be used later, in (B12).

Again due to the block form of $A_{T}$ as in (B1),

$$
T^{-T} M T^{-1}=A_{T}^{T}\left(A_{T} P_{T} A_{T}^{T}+Q_{T}\right)^{-1} A_{T}=\left[\begin{array}{cc}
\Lambda^{T} \Pi \Lambda & 0 \\
0 & 0
\end{array}\right]
$$

where $\Pi$ is the first $r \times r$ diagonal block of $\left(A_{T} P_{T} A_{T}^{T}+Q_{T}\right)^{-1}$. By applying Lemma 7 to the right hand side of (B9),

$$
\Pi=\left[\Lambda P_{T}^{(1,1)} \Lambda^{T}+Q_{T}^{(1,1)}-Q_{T}^{(1,2)}\left(Q_{T}^{(2,2)}\right)^{-1} Q_{T}^{(2,1)}\right]^{-1} .
$$

By assumption $Q \geq 0$, then $Q_{T} \geq 0$. Apply Lemma 10 to $Q_{T}$, then

$$
Q_{T}^{(1,1)}-Q_{T}^{(1,2)}\left(Q_{T}^{(2,2)}\right)^{-1} Q_{T}^{(2,1)} \geq 0
$$


hence

$$
\Lambda P_{T}^{(1,1)} \Lambda^{T}+Q_{T}^{(1,1)}-Q_{T}^{(1,2)}\left(Q_{T}^{(2,2)}\right)^{-1} Q_{T}^{(2,1)} \geq \Lambda P_{T}^{(1,1)} \Lambda^{T}>0 .
$$

Lemma 8 then leads to

$$
\Pi=\left[\Lambda P_{T}^{(1,1)} \Lambda^{T}+Q_{T}^{(1,1)}-Q_{T}^{(1,2)}\left(Q_{T}^{(2,2)}\right)^{-1} Q_{T}^{(2,1)}\right]^{-1} \leq\left[\Lambda P_{T}^{(1,1)} \Lambda^{T}\right]^{-1} .
$$

Recalling (B11) then yields

$$
T^{-T} M T^{-1} \leq\left[\begin{array}{cc}
\left(P_{T}^{(1,1)}\right)^{-1} & 0 \\
0 & 0
\end{array}\right]
$$

Now apply Lemma 11 to $P_{T}$, then

$$
\begin{gathered}
T^{-T} M T^{-1} \leq P_{T}^{-1}, \\
M \leq T^{T} P_{T}^{-1} T=P^{-1} .
\end{gathered}
$$

Inequality (31) is then established.

\section{References}

Anderson, B. D. O. and Moore, J. B. (1979). Optimal Filtering. Prentice-Hall, Inc., New Jersey.

Deyst Jr, J. J. (1973). Correction to "Conditions for asymptotic stability of the discrete minimumvariance linear estimator". IEEE Transactions on Automatic Control, 18(5):1973.

Deyst Jr, J. J. and Price, C. F. (1968). Conditions for asymptotic stability of the discrete minimumvariance linear estimator. IEEE Transactions on Automatic Control, 13(6):702-705.

Grewal, M. S. and Andrews, A. P. (2015). Kalman Filtering: Theory and Practice Using Matlab. WileyIEEE Press, 4th edition.

Ioannou, P. and Sun, J. (1996). Robust Adaptive Control. Prentice Hall. http://wwwrcf.usc.edu/ ioannou/Robust_Adaptive_Control.htm.

Jazwinski, A. H. (1970). Stochastic Processes and Filtering Theory, volume 64 of Mathematics in Science and Engineering. Academic Press, New York and London.

Kalman, R. E. (1963). New methods in Wiener filtering theory. In Bogdanoff, J. L. and Kozin, F., editors, Proceedings of the First Symposium on Engineering Applications of Random Function Theory and Probability, New York. John Wiley \& Sons.

Kim, P. (2011). Kalman Filter for Beginners: with Matlab Examples. CreateSpace.

Ljung, L. (1999). System Identification - Theory for the User. Prentice-Hall, 2nd edition edition.

Moore, J. B. and Anderson, B. D. O. (1980). Coping with singular transition matrices in estimation and control stability theory. International Journal of Control, 31(3):571-586.

Sastry, S. and Bodson, M. (1989). Adaptive Control: Stability, Convergence and Robustness. PrenticeHall.

Tóth, R. (2010). Modeling and Identification of Linear Parameter-Varying Systems. Springer.

Zarchan, P. and Musof, H. (2005). Fundamentals of Kalman filtering: a practical approach. AIAA.

Zhang, L. and Zhang, Q. (2015). Observability conservation by output feedback and observability Gramian bounds. Automatica, 60:38-42.

Zhang, Q. (2017). On stability of the Kalman filter for discrete time output error systems. Systems and Control Letters, 107:84-91.

Zhang, Q. and Besançon, G. (2008). An adaptive observer for sensor fault estimation in a class of uniformly observable non-linear systems. International Journal of Modelling, Identification and Control, $4(1): 37-43$. 